\title{
RESIDUE RINGS OF SEMI-PRIMARY HEREDITARY RINGS*
}

\begin{abstract}
ABRAHAM ZAKS
Introduction: Throughout this paper we assume that all rings contain an identity. We say that $R$ is a semi-primary ring if its (Jacobson) radical $N$ is nilpotent, and $R / N$ is an Artinian ring. We say that $R$ admits a splitting, and we write $R=A+B$ if $A$ is a subring of $R$, if $B$ is a two-sided ideal in $R$, and if $A \cap B=0$.

It has been shown in [1] that for a semi-primary $\operatorname{ring} R \quad l \cdot g l \cdot \operatorname{dim} R$ $=r \cdot g l . \operatorname{dim} R=1+l \cdot$ proj. $\operatorname{dim} N$. This common value is denoted by $g l \cdot \operatorname{dim} R$.

It has been shown in [2] that if $R$ is a semi-primary hereditary ring, and $\mathrm{I}$ is a two-sided ideal in $R$, then $g l \cdot \operatorname{dim} R / I<\infty$.

We prove that if $R$ is a semi-primary ring and $g l \cdot \operatorname{dim} R / N^{2}<\infty$, then $R$ is a residue ring of a semi-primary hereditary ring. This is a generalization of a similar result in [3]. The crucial step is a splitting theorem that we prove for a semi-primary ring $R$, for which $e N e=0$ for any primitive idempotent $e \in R$. This splitting theorem seems also useful in studying certain types of semiprimary subrings of a simple ring.

The author wishes to thank Professors M. Auslander, E.E. Lazerson, and M.I. Rosen for their helpful remarks and suggestions in the preparation of this paper.
\end{abstract}

\section{§1. A Splitting Theorem.}

For the rest of this section, let $R=\sum_{u=1}^{t} R e_{u}$ be a complete decomposition for the semi-primary ring $R$, i.e. $e_{1} \ldots \ldots e_{t}$ are primitive orthogonal idempotents (e.g. [4, pp 53-57]). Furthermore, assume $e_{v} N e_{v}=0$ for $v=1, \ldots \ldots, t$. When writing $e_{i}, e_{j} \ldots .$. we always assume $1 \leq i, j, \ldots \ldots \leq t$, unless otherwise stated.

Since for any $e_{i}, e_{i} N e_{i}$ is the radical of $e_{i} R e_{i}$, and $e_{i} R e_{i} / e_{i} N e_{i}$ is a division ring, we have:

Received July 8, 1966.

This paper is based on a part of the author's doctoral dissertation written at Brandeis University under the direction of Professor Maurice Auslander. 
Lemma 1: $\quad e_{i} R e_{i}$ is a division ring for $i=1, \ldots . ., t$. Every element $e_{i} r e_{j} \in R$ induces a homomorphism (by right multiplication) of $R e_{i}$ into $R e_{j}$, and vice versa. In particular, if $R e_{i}$ is isomorphic to $R e_{j}$, then $e_{k} N e_{i} \neq 0$ iff $e_{k} N e_{j} \neq 0$ for any $k, 1 \leq k<t$. Thus:

Lemma 2: Let $R e_{i}$ be isomorphic to $R e_{j}$, then $e_{i} N e_{j}=0$.

One easily verifies that this is equivalent to:

Lemma 2*: Every non-zero homomorphism between isomorphic components is an isomorphism.

Let $\Gamma_{0}$ be $\sum_{i, j} e_{i} R e_{j}$ where $(i, j)$ ranges over all pairs such that $R e_{i}$ and $R e_{j}$ are isomorphic to $R e_{i_{0}}$ for some fixed $i_{0}$. Let $R_{0}=\operatorname{Hom}_{R}\left(\sum_{k} R e_{k}, \sum_{k} R e_{k}\right)$, where $k$ ranges over all indices such that $R e_{k}$ is isomorphic to $R e_{i_{0}}$. Let $R_{1}=$ $\operatorname{Hom}_{R}\left(\sum_{i=1}^{s} B_{i}, \sum_{i=1}^{s} B_{i}\right)$, where $B_{i}=R e_{i_{0}}$ for $i=1, \ldots \ldots, s$, and $s$ is the number of components in the complete decomposition for $R$ which are isomorphic to $R e_{i_{0}}$. Finally, let $\Gamma_{1}=\left(e_{i_{0}} R e_{i_{0}}\right)_{s}$-the $s \times s$ matrix algebra over the division ring $e_{i_{0}} R e_{i_{0}}$. With these notations we have:

Lemma 3: The subring $\Gamma_{0}$ of $R$ is a simple ring.

Proof: It is clear that $R_{1}$ and $R_{0}$ are isomorphic. It is also clear that $\Gamma_{0}\left(\Gamma_{1}\right)$ is anti-isomorphic to $R_{0}\left(R_{1}\right)$. Thus $\Gamma_{0}$ and $\Gamma_{1}$ are isomorphic.

Let $\Gamma$ be $\sum_{i, j} e_{i} R e_{j}$, where $(i, j)$ ranges over all pairs such that $R e_{i}$ is isomorphic to $R e_{j}$. Since on $\Gamma$ we have a natural splitting, into subsums taken over any fixed isomorphism class of components, it follows from Lemma 3 that:

Proposition 1: The subring $\Gamma$ of $R$ is a semi-simple ring.

The underlying additive group of $R$ admits a decomposition $R=\sum_{i, j=1}^{t} e_{i} R e_{j}$. Let $R_{1}=\sum_{i, j} e_{i} R e_{j}$ where $(i, j)$ ranges over all pairs such that $R e_{i}$ is not isomorphic to $R e_{j}$. We have $R=\Gamma+R_{1}$, and it is clear that $R_{1} \subset N$. Our next step is to show that $R_{1}=N$. We will be done once we show that $R_{1}$ is a two-sided ideal in $R$. Since $r=\sum_{i, j=1}^{t} e_{i} r e_{j}$ for any $r \in R$, and since $R_{1}$ is closed under addition, it suffices to show that $e_{i} r e_{j} \in R_{1}$, implies $e_{i} r e_{j} s e_{k} \in R_{1}$ and $e_{l} v e_{i} r e_{j} \in R_{1}$ for all $1 \leq i, j, k, l \leq t$ and $r, s, v \in R$. But $e_{i} r e_{j} s e_{k} \notin R_{1}$ only if $R e_{i}$ is isomorphic to $R e_{k}$, whence by Lemma $2^{*}$ this element induces an isomorphism of $R e_{i}$ onto $R e_{k}$, and this is impossible since $e_{i} r e_{j} \in R_{1} \subset N$. A similar argument shows that $e_{\imath} v e_{i} r e_{j} \in R_{1}$. 
This proves:

Theorem 1. The Splitting Theorem: Let $R$ be a semi-primary ring, and let $R=\sum_{u=1}^{t} R e_{u}$ be a complete decomposition for $R$. If $e_{i} N e_{i}=0$ for $i=1, \ldots$ $\ldots, t$, then $R$ admits a splitting $R=\Gamma+N . \Gamma=\sum_{i, j} e_{i} R e_{j}$ where $(i, j)$ ranges over all pairs such that $R e_{i}$ is isomorphic to $R e_{j} . \quad N=\sum_{i, j} e_{i} R e_{j}$ where $(i, j)$ ranges over all pairs such that $R e_{i}$ is not isomorphic to $R e_{j}$.

With the assumptions and notations of Theorem 1, using Lemma 1 one can easily prove that the center of $R$ is a direct product of fields. The center of $R$ is a field only if 0 and 1 are the unique central idempotents in $R$. One can also show that if $R=\Gamma_{1}+N$ is another splitting for $R$, then there exists an invertible element $s$ in $R$ such that the automorphism $r \rightarrow s r s^{-1}$ takes $\Gamma$ onto $\Gamma_{1}$.

The splitting theorem enables us to view $N$ as a $\Gamma-\Gamma$ bimodule. Define $\Omega(\Gamma, N)=\sum_{i=0}^{\infty} N^{(\imath)}$, where $N^{(0)}=\Gamma$ and $N^{(\imath)}=N^{(i-1)} \underset{\Gamma}{\otimes} N$. Letting $n_{1} \otimes \ldots \ldots \otimes n_{i} \otimes n^{1}$ $\otimes \ldots \ldots \otimes n^{j}=n_{1} \otimes \ldots \ldots \otimes n_{i} \otimes n^{1} \otimes \ldots \ldots \otimes n^{j}$ and extending $\otimes$ distributively, $\Omega(\Gamma, N)$ becomes a ring (identifying $N^{(i)} \underset{\Gamma}{\otimes}, \Gamma \underset{\Gamma}{\otimes} N^{(i)}$ and $N^{(i)}$ for $\left.i \geq 0\right)$. Letting $f\left(n_{1}\right.$ $\left.\otimes \ldots \ldots \otimes n_{k}\right)=n_{1} \ldots \ldots \cdot n_{k}$, and extending $f$ linearly, $f$ is a ring epimorphism from $\Omega(\Gamma, N)$ onto $R$. If for some $m, N^{(m)}=0$ then $M=\sum_{i=1}^{m-1} N^{(i)}$ is a nilpotent two-sided ideal and $\Omega(\Gamma, N) / M$ is semi-simple. Thus $\Omega(\Gamma, N)$ is a semi-primary ring with radical $M$. Furthermore, $M=\Omega(\Gamma, N) \underset{\Gamma}{\otimes}$, and since $N$ is $\Gamma$-projective, $M$ is $\Omega(\Gamma, N)$-projective. By [1], this implies that $\Omega(\Gamma, N)$ is an hereditary ring.

If $E_{0}, \ldots \ldots, E_{k}$ are primitive idempotents in $R$, then $\left(E_{0}, \ldots \ldots, E_{k}\right)$ is an $R$ connected sequence of length $k$ if $E_{i} N E_{i+1} \neq 0$ for $i=0, \ldots \ldots, k-1$. It is obvious that $N^{(m)}=0$ if there are no $R$-connected sequences of length $m$.

2. Applications. We first deal with the case $g l \cdot \operatorname{dim} R / N^{2}<\infty$. Thus let $R$ be a semi-primary ring and $g l \cdot \operatorname{dim} R / N^{2}<\infty$. Let $\tilde{R}=R / N^{2}, \tilde{N}=N / N^{2}$. With the notations of section 1 we have that $\tilde{R}=\sum_{u=1}^{t} \widetilde{R} \tilde{e}_{u}$ is a complete decomposition for $\tilde{R}$, where $\tilde{e}_{i}$ is the canonical image of $e_{i}$ in $\tilde{R}$ for $i=1, \ldots \ldots, t$. By a result in [3] concerning semi-primary rings for which the square of the radical is zero, we conclude that $\tilde{R}$-connected sequences are bounded in length. This implies:

Lemma 4: R-connected sequences are bounded in length.

Proof: We show that if $e_{i} N e_{j} \neq 0$ then there exists an $\tilde{R}$-connected sequence 
of the form $\left(\tilde{e}_{i}, \ldots \ldots, \tilde{e}_{j}\right)$. If $\left(\tilde{e}_{i}, \tilde{e}_{j}\right)$ is $\tilde{R}$-connected we are done. Otherwise, $e_{i} N e_{j} \in N^{2}$ and there readily follows the existence of a primitive idempotent $e_{k}$ such that $e_{i} N e_{k} N e_{j} \neq 0$. If $\left(\tilde{e}_{i}, \tilde{e}_{k}, \tilde{e}_{j}\right)$ is $\widetilde{R}$-connected we are done. Otherwise, either $e_{i} N e_{k} \in N^{2}$ or $e_{k} N e_{j} \in N^{2}$. Let $e_{i} N e_{k} \in N^{2}$, then we can find a primitive idempotent $e_{l}$ such that $0 \neq e_{i} N e_{l} N e_{k} N e_{j} \in N^{3}$. Since $N$ is nilpotent, this procedure must end and the result follows.

In particular, we must have $e_{i} N e_{i}=0$ for $i=1, \ldots \ldots, t$, thus by Theorem 1 , $R=\Gamma+N$. The ring $\Omega(\Gamma, N)$ as constructed at the end of $\S 1$ is a semi-primary hereditary ring in this case. Combining this with the result in [2] concerning residue rings of semi-primary hereditary rings we have:

TheORem 2. Let $R$ be a semi-primary ring, then the following are equivalent:

(a) $R$ is a residue ring of a semi-primary hereditary ring.

(b) All residue rings of $R$ have finite global dimension.

(c) $g l \cdot \operatorname{dim} R / N^{2}<\infty$.

Remark that under each of these equivalent conditions $e N e=0$ for any primitive idempotent $e \in R$.

In particular, if $R$ is a semi-primary hereditary ring, its center is a direct product of fields. The center of $R$ is a field only if 0 and 1 are the unique central idempotents in $R$.

For the rest, let $D$ be a division ring and let $D_{n}$ denote the $n \times n$ matrix algebra over $D$. Let $R$ be a semi-primary subring of $D_{n}$, such that $R=\sum_{i=1}^{n} R e_{i}$ is a complete decomposition for $R$. Without loss of generality we may assume that $e_{i}$ is the matrix whose $(\alpha, \beta)^{t h}$ component is $\left(e_{i}\right)_{\alpha \beta}=\delta_{i \alpha} \delta_{i \beta}$ for all $i, \alpha, \beta=1, \ldots$ $\ldots, n$. We can (naturally) identify $e_{i} D_{n} e_{i}$ with $D$, and $e_{i} R e_{i}$ with a subring of $D$, for $i=1, \ldots \ldots, n$. In particular $e_{i} N e_{i}=0$ for $i=1, \ldots \ldots, n$, and by Theorem 1 $R=\Gamma+N$. We want to show now that $\Omega(\Gamma, N)$ is a semi-primary hereditary ring. This follows from the fact that any element $e_{i} r e_{j} \in R$ induces an isomorphism from $D_{n} e_{i}$ onto $D_{n} e_{j}$. Thus in particular $e_{i} r e_{j} \neq 0$ and $e_{k} s e_{i} \neq 0$ imply $e_{k} s e_{i} r e_{j} \neq 0$, or $e_{i} N e_{j} \neq 0$ and $e_{j} N e_{k} \neq 0$ imply $e_{i} N e_{j} N e_{k} \neq 0$. Since $N$ is nilpotent this implies that $R$-connected sequences are bounded in length. Thus we proved:

Theorem 3. Let $R$ be a semi-primary subring of $D_{n}$, containing $n$ orthogonal idempotents, then $\mathrm{gl} \cdot \mathrm{dim} R / I<\infty$ for any two-sided ideal $I$ in $R$.

Let $R$ be a semi-primary subring of $D_{n}$. Let $C(R)$ be the subset of $D_{n}$ 
consisting of elements $V \in D_{n}$ for which $V r=r V$ for all $r \in R$. Set $C\left(D_{n}\right)$ to be the center of $D_{n}$. One can show that $C(R)=C\left(D_{n}\right)$ implies that (a) 0 and 1 are the unique central idempotents in $R$ and (b) $R$ contains $n$ orthogonal idempotents. If $D$ is a field one easily verifies that (a) and (b) imply $C(R)=C\left(D_{n}\right)$.

\section{REFERENCES}

[1] M. Auslander-On the Dimension of Modules and Algebras III, Global Dimension, Nagoya Math, J., 9(1955), pp. 67-77.

[2] S. Eilenberg, H. Nagao and T. Nakoyama-On the Dimension of Modules and Algebras IV, Dimension of Residue Rings of Hereditary Rings, Nagoya Math. J., 10 (1956), pp 87-95

[3] J.P. Jans and T. Nakayama-On the Dimension of Modules and Algebras VIII, Algebras with Finite Dimensional Residue Algebras, Nagoya Math. J., 11 (1957), pp 67-76.

[4] N. Jacobson-Structure of Rings, Amer. Math. Soc. Col. Pub., vol. 37 (1956).

Brandeis University Waltham, Massachusetts 